' Disciplina de Endocrinologia e Metabologia, Faculdade de Medicina de São José do Rio Preto, São José do Rio Preto, SP, Brasil
Correspondência para: Daniel Laguna Neto

Rua Rosa Scavazza, 715 - Jardim Renascença

15130-000 - Mirassol, SP, Brasil

dlagunaneto@yahoo.com.br

Recebido em 7/Jan/2009

Aceito em 8/Fev/2009

\section{Avaliação da glicemia capilar na ponta de dedo versus locais alternativos - Valores resultantes e preferência dos pacientes}

\author{
Analysis of fingerstick capillary glycemia versus \\ alternative site - Results and patients preferences
}

Daniel Laguna Neto', Fernando César Robles ${ }^{1}$, Fábio Guirado Dias ${ }^{1}$, Antônio Carlos Pires ${ }^{1}$

\begin{abstract}
RESUMO
O trabalho visa avaliar eficácia e a preferência dos pacientes na monitorização da glicemia capilar em locais alternativos: lóbulo de orelha, antebraço e panturrilha em relação à ponta de dedo. Foram avaliados 89 pacientes $(39 \mathrm{M} / 50 \mathrm{~F}$ ) com diabetes melito tipo 2 (DM2). A monitorização foi feita com glicosímetro digital (ACCU - CHEK Performa, Roche), lancetador ACCU-CHEK Multiclix, em grau 5 na face palmar da falange distal do $3^{\circ}$ dedo da mão direita, porção inferior do lóbulo da orelha direita, antebraço direito e região superior da panturrilha direita. Não houve diferença da glicemia capilar na ponta de dedo em relação aos locais alternativos. A ponta de dedo foi o local mais doloroso. Quanto ao local de preferência, a orelha e a ponta de dedo foram os preferidos. A monitorização glicêmica em locais alternativos é simples, segura e eficaz, como a glicemia de ponta de dedo. Arq Bras Endocrinol Metab. 2009;53(3):344-7.
\end{abstract}

Descritores

Glicemia capilar; locais alternativos; diabetes melito; monitorização glicêmica

\begin{abstract}
This paper evaluate the efficacy and patients preferences of glucose monitoring in alternatives sites: ear lobe, forearm and calf against conventional fingerstick. We studied 89 patients (39 M/ $50 \mathrm{~F}$ ) with type 2 diabetes mellitus (DM2). Glucose monitoring was measured using a digital glucosimeter (ACCU - CHEK Performa, Roche), and ACCU-CHEK Multiclix lancetador, with grade 5 on the distal phalange on the right hand's third finger, inferior part of right ear lobe, right forearm and right calf. There was not statistical significance on the comparative analyzes of fingerstick and alternatives sites. There was more pain in fingerstick. The patients preferred the fingerstick and the ear lobe. Glucose monitoring in alternative sites is as simple, safe and efficient as fingerstick. Arq Bras Endocrinol Metab. 2009;53(3):344-7.
\end{abstract}

Keywords

Capillary glycemia; alternative sites; diabetes mellitus; blood glucose monitoring
$\mathrm{O}$ diabetes melito tipo 2 (DM2) é uma doença crônica que acomete milhões de pessoas em todo o mundo e sua prevalência aumenta consideravelmente, ano após ano (1). De acordo com a Organização Mundial da Saúde (OMS) e a Internacional Diabetes Federation (IDF), o número de diabéticos no mundo dobrará para 366 milhões em 2030, dos 171 milhões na atualidade. Somente no município de São José do Rio Preto,
SP, estima-se que haja em torno de 16 mil pacientes, ou seja, $4,27 \%$ da população (2).

O controle intensivo do DM2, conforme foi demonstrado no United Kingdon Prospective Diabetes Stu$d y$ (UKPDS) (3), posteriormente o STENO-2 Study (4) e na publicação dos dez anos de seguimento do UKPDS (5), é essencial para prevenir ou retardar as complicações crônicas da doença. O monitoramento do DM2, por 
meio da glicemia capilar, é um dos alicerces do controle intensivo. Apesar disso, aproximadamente, 23\% dos pacientes deixam de realizar a automonitorização "nas pontas dos dedos" (6) por causa da dor decorrente das inúmeras terminações nervosas locais.

Em busca de conforto para o paciente, a glicemia capilar em locais alternativos tem sido estudada, entretanto, estima-se que os valores encontrados nesses locais possam diferir dos realizados na ponta de dedo (7).

Atualmente, existem poucas evidências sobre o uso de locais alternativos para a automonitorização. Já foram realizados estudos utilizando o braço, o antebraço (8) e o lóbulo da orelha (9), comparando com a ponta do dedo. Não foi encontrado nenhum trabalho que comparasse diferentes locais alternativos entre si e com a ponta do dedo.

O propósito deste estudo foi avaliar a eficácia e a preferência dos pacientes em relação aos locais alternativos pré-determinados: lóbulo de orelha, antebraço e panturrilha, comparada à ponta de dedo.

\section{MÉTODOS}

Foi realizado um estudo comparativo, prospectivo, com análise quantitativa e qualitativa, de pacientes com DM2 atendidos no Ambulatório de Diabetes da Disciplina de Endocrinologia e Metabologia da Faculdade de Medicina de São José do Rio Preto (Famerp), SP, durante o período de agosto a dezembro de 2008. Este trabalho foi submetido e aprovado pelo Comitê de Ética em Pesquisa em Seres Humanos da Famerp sob o no ${ }^{-}$155/2008.

Foram avaliados 89 pacientes com DM2, sendo 39 $(43,9 \%)$ do sexo masculino e $50(56,1 \%)$ do sexo feminino. A idade variou de 18 a 85 anos, sendo a média de 59,4 \pm 12 anos. As variáveis estudadas foram idade, sexo, glicemia em ponta de dedo, glicemia nos locais alternativos, dor por ocasião da aplicação e método de preferência. Foram excluídos os seguintes pacientes: pacientes com neuropatia diabética preestabelecida ou com amputações de membros que impossibilitassem a avaliação dos locais alternativos, pacientes em uso de analgésico e anti-inflamatório potentes, discrasia sanguínea ou uso de anticoagulante.

A monitorização foi realizada em jejum, por uma técnica em enfermagem, em ambiente ambulatorial, com glicosímetro digital (ACCU - CHEK Performa, Roche), lancetador ACCU-CHEK Multiclix e respectivas lancetas, graduado de 1 a 5 em graus crescentes de profundidade de penetração na pele. Foi padronizado o grau 5 para realização do trabalho. As medidas foram realizadas na face palmar da falange distal do $3^{\circ}$ dedo da mão direita, porção inferior do lóbulo da orelha direita, antebraço direito e região superior da panturrilha direita, de maneira simultânea, para efeito de comparação.

A avaliação da intensidade da dor fundamentou-se na informação do próprio paciente. A sensação dolorosa foi graduada em A (ausente), B (muito pouco), C (pouco), D (moderada) e E (intensa).

A preferência do local teve como base a informação do paciente após o término das medidas nos locais determinados.

Os dados foram coletados em uma ficha padrão e analisados pelo software Minitab, por meio do teste quiquadrado, teste te teste de correlação de concordância de Lin. Os pacientes foram incluídos no estudo após assinatura do termo de consentimento livre e esclarecido.

\section{RESULTADOS}

Foram avaliados 89 pacientes com medida da glicemia na ponta de dedo e nos locais alternativos. Em apenas um deles não foi possível medir a glicemia no antebraço por dificuldade técnica.

Em relação aos resultados da glicemia capilar, não houve diferença estatisticamente significativa entre a glicemia avaliada na ponta de dedo e a glicemia avaliada nos demais locais alternativos (Tabela 1 e Figuras 1, 2 e 3).

\begin{tabular}{|c|c|c|c|c|c|c|}
\hline Local do exame & $\mathbf{n}$ & $\begin{array}{c}\text { Glicemia }(\mathrm{mg} / \mathrm{dL}) \\
\text { Média }\end{array}$ & $\begin{array}{c}\text { Glicemia (mg/dL) } \\
\text { DP }\end{array}$ & $\begin{array}{c}\text { Glicemia }(\mathrm{mg} / \mathrm{dL}) \\
\text { Mediana }\end{array}$ & $\underset{\text { Variação }}{\text { Glicemia }(m g / d L)}$ & $T / p$ \\
\hline Ponta de dedo & 89 & 174,9 & 87,3 & 156,0 & $51-467$ & \\
\hline Lóbulo de orelha & 89 & 170,9 & 82,9 & 148,0 & $51-457$ & $\begin{array}{c}T=1,57 \\
p=0,121\end{array}$ \\
\hline Antebraço & 88 & 172,2 & 86,2 & 148,0 & $51-451$ & $\begin{array}{c}T=1,76 \\
p=0,082\end{array}$ \\
\hline Panturrilha & 89 & 168,5 & 80,8 & 146,0 & $53-462$ & $\begin{array}{c}T=1,94 \\
p=0,064\end{array}$ \\
\hline
\end{tabular}




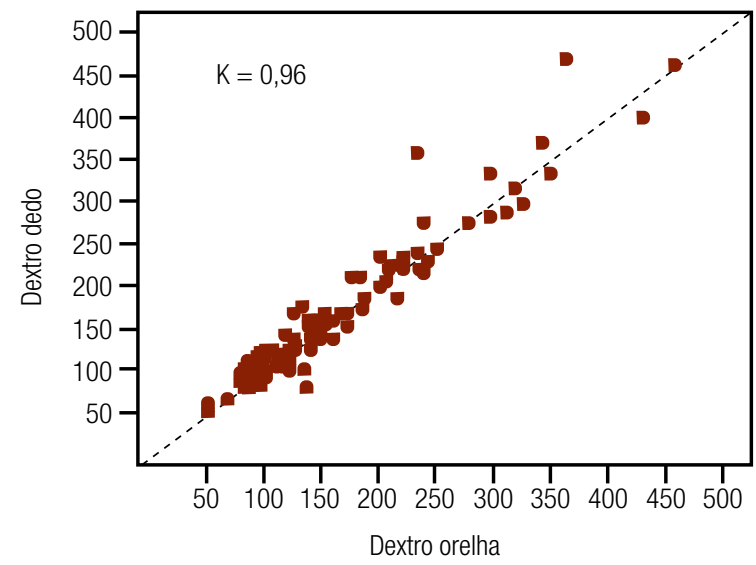

Figura 1. Análise da correlação de concordância entre a glicemia capilar na ponta de dedo e na orelha.

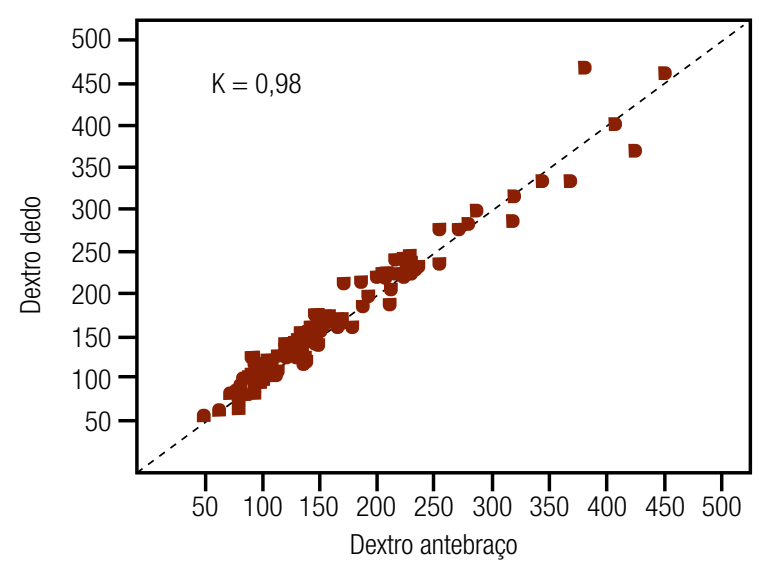

Figura 2. Análise da correlação de concordância entre a glicemia capilar na ponta de dedo e no antebraço.

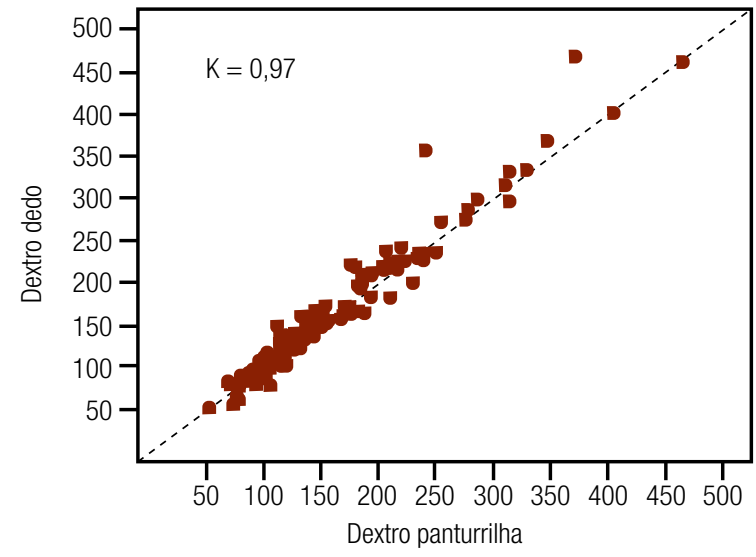

Figura 3. Análise da correlação de concordância entre a glicemia capilar na ponta de dedo e na panturrilha.
Quanto à dor durante o exame, nos exames realizados na orelha, no antebraço e na panturrilha, verificouse que, respectivamente, $79 \%, 71 \%$ e $73 \%$ dos pacientes referiram nenhum desconforto, ao passo que apenas $48 \%$ dos pacientes não apresentaram desconforto no exame realizado na ponta de dedo (Figura 4). Porém, não houve correlação estatística significativa entre a dor no exame realizado no dedo quando comparado aos demais. Em relação ao local de escolha, $32(36 \%)$ pacientes preferiram o dedo, $32(36 \%)$ escolheram a orelha, $13(14,5 \%)$ optaram pela panturrilha e $12(13,5 \%)$ optaram pelo antebraço. Não houve diferença estatística significativa na escolha quando analisado por sexo $(\mathrm{p}$ $=0,57)$ e idade do paciente $(\mathrm{p}=0,17)$.

\section{DISCUSSÃO}

Analisando os resultados deste estudo, pode-se avaliar a eficácia da monitorização da glicemia nos locais alternativos.

Uma das grandes preocupações médicas é a precisão da glicemia nos locais alternativos. Ferraz, Maia e Araújo (9) demonstraram que não há diferença da glicemia medida na ponta de dedo, quando comparado à orelha. No presente estudo, os resultados foram semelhantes aos dados de literatura. Não houve diferença significativa da glicemia medida na ponta de dedo, quando comparada à glicemia medida em orelha, antebraço e panturrilha.

Avaliando o local de escolha, o sexo e a idade, não houve influência. O dedo e a orelha foram os locais preferidos, apesar de os pacientes referirem maior sensação dolorosa na ponta de dedo em comparação aos locais alternativos. Tal fato talvez se deva ao hábito da monitorização ser sempre realizada na ponta de dedo.

Um dado que chamou a atenção para utilizar os pontos alternativos seria a dificuldade técnica em realizar o exame. Viana e cols. (10) demonstraram que o déficit visual dificultaria a monitorização nos locais alternativos. No presente estudo, não houve como avaliar dificuldade técnica, pois as medidas foram realizadas por uma auxiliar de enfermagem. Seria necessário estudo com automonitorização para avaliar a dificuldade técnica.

A glicemia capilar nos locais alternativos é alternativa simples e sem diferença significativa em relação à glicemia na ponta de dedo e, portanto, pode ser uma opção para melhorar a adesão dos pacientes ao tratamento e, desse modo, reduzir o risco de complicações micro e macrovasculares do DM2.

Agradecimentos: Agradecemos ao Prof. Dr. José Antonio Cordeiro pela ajuda nas análises dos dados e à Roche pelo fornecimento do material. 


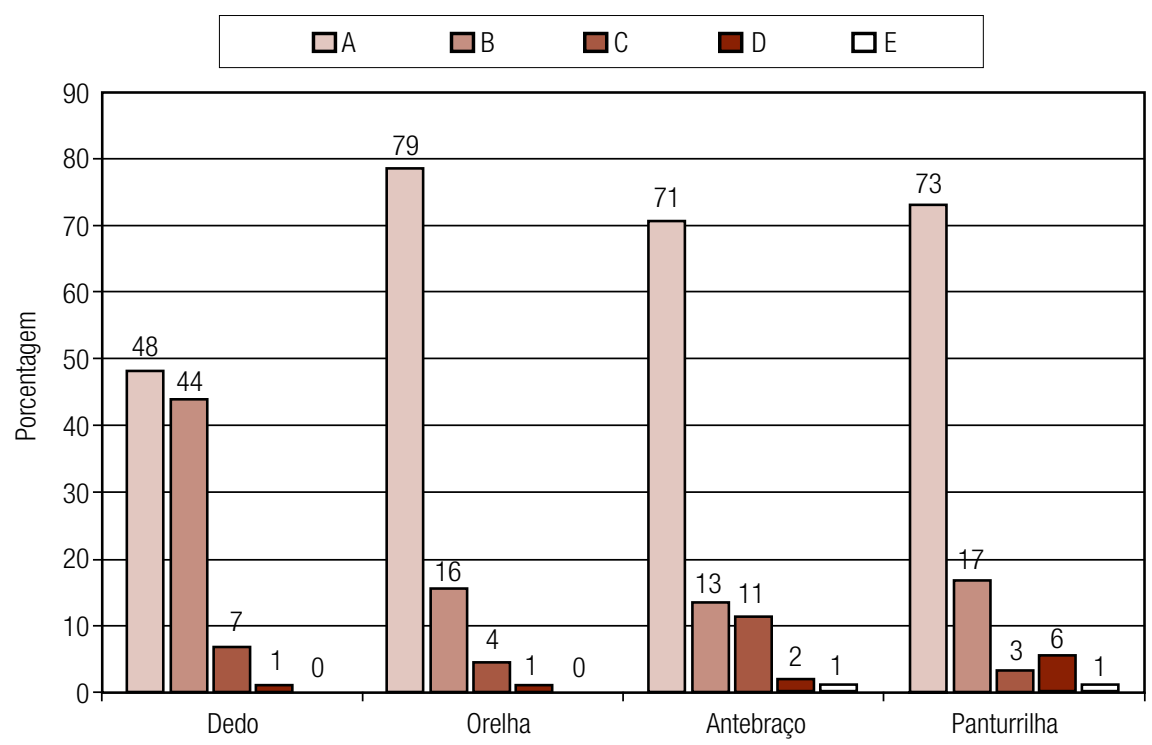

Figura 4. Análise da dor durante 0 exame nos diferentes locais $(A=$ ausente, $B=$ muito pouco, $C=$ pouco, $D=$ moderada e $E=$ intensa $)$.

Declaração: os autores declaram não haver conflitos de interesse científico neste estudo.

\section{REFERÊNCIAS}

1. American Diabetes Association. Standards of medical care for patients with diabetes mellitus (position statement). Diabetes Care. 2008;31 Suppl 1:S12-54.

2. HiperDia. Disponível em www.hiperdia.datasus.gov.br.

3. Intensive blood-glucose control with sulphonylureas or insulin compared with conventional treatment and risk of complications in patients with type 2 diabetes (UKPDS 33): UK Prospective Diabetes Study (UKPDS) Group. Lancet. 1998;352:837-53.

4. Gaede P, Lund-Andersen $\mathrm{H}$, Parving HH, Pedersen O. Effect of a multifactorial intervention on mortality in type 2 diabetes. $\mathrm{N}$ Engl J Med. 2008;358:580-591.
5. Holman RR, Paul SK, Bethel MA, Matthews DR, Neil HA. 10-year follow-up of intensive glucose control in type 2 diabetes. N Engl J Med. 2008;359:1577-89.

6. Burge MR. Lack of compliance with home blood glucose monitoring predicts hospitalization in diabetes. Diabetes Care. 2001;24:1502-3.

7. Peled N, Wong D, Gwalani SL. Comparison of glucose levels in capillary blood samples obtained from a variety of body sites. Diabetes Technol Ther. 2002;4:35-44.

8. Fedele D, Corsi A, Noacco C, Prisco F, Squatrito S, Torre E, et al. Alternative site blood glucose testing: a multicenter study. Diabetes TechnolTher. 2003;5(6):983-9.

9. Ferraz DP, Maia FF, Araújo LR. [Fingerstick capillary glycemia versus ear lobe measurement: comparative analysis of results and patient preferences]. Arq Bras Endocrinol Metabol. 2004;48:389-93.

10. Viana AGD, Lins $D$, Jacob RS, Liberman $B$. [Locais alternativos de coleta de sanguínea na auto-monitorização da glicemia - um estudo comparativo]. Arq Bras Endocrinol Metabol. 2003;47/2:S179. 